\title{
Consolidando la calidad en las bibliotecas universitarias: evaluaciones, sellos, diplomas y certificaciones
}

\author{
Por Núria Balagué
}

\begin{abstract}
Resumen: La construcción efectiva del Espacio europeo de educación superior se ha convertido en un dinamizador de muchas actividades relacionadas con la calidad y la adopción de sistemas de garantía de la calidad en el seno de las universidades $y$, por ende de sus bibliotecas; es una decisión inaplazable. Un tercio de las bibliotecas universitarias españolas dispone de algún tipo de reconocimiento público de la calidad de su gestión. Se presentan las diversas vías que han seguido las bibliotecas universitarias para disponer de este reconocimiento: certificaciones, diplomas y sellos de calidad y se constata la existencia de una cierta tendencia a la multicertificación.
\end{abstract}

Palabras clave: Gestión de la calidad, Bibliotecas universitarias, EFQM, ISO 9001.

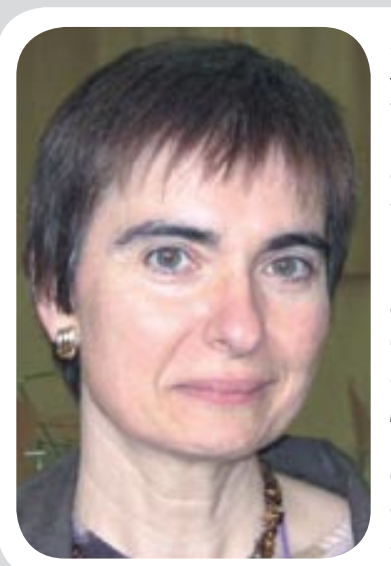

Núria Balagué es subdirectora del Servicio de Bibliotecas de la Universidad Autónoma de Barcelona. También colabora como profesora asociada con la Facultad de Biblioteconomía y Documentación de la Universidad de Barcelona, donde imparte asignaturas relacionadas con la planificación y la gestión de la calidad. Es doctora en biblioteconomía y documentación por la Universidad de Barcelona. Ha formado parte del Comité de evaluación de Aneca del Certificado de Calidad de los Servicios de Biblioteca de las Universidades.

\section{Title: Quality improvement in university libraries: evaluations, quality seals, diplomas and cer- tifications}

\begin{abstract}
The effective construction of the European Higher Education Area (EHEA) has given rise to many activities related to quality and the adoption of quality assurance systems in universities and their libraries can no longer be postponed. One third of Spanish university libraries have gained some type of public recognition for their system of quality management. The variety of approaches followed by university libraries to achieve this recognition is presented: certifications, diplomas and quality seals; a certain trend toward multi-certification is described.
\end{abstract}

Keywords: Quality management, University libraries, EFQM, ISO 9001.

Balagué, Núria. "Consolidando la calidad en las bibliotecas universitarias: evaluaciones, sellos, diplomas y certificaciones”. En: El profesional de la información, 2007, julio-agosto, v. 16, n. 4, pp. 338-342.

DOI: 10.3145/epi.2007.jul.06

\section{Introducción}

\section{LA INTERNACIONALIZA-} CIÓN ES UNO de los factores que ha llevado al sector de la educación superior a ver la necesidad de garantizar la calidad de sus procesos y actividades. Los gobiernos, la explosión de la exportación educativa, la competencia entre universidades $y$ el peligro del aislamiento, presionan a las instituciones de enseñanza para que sean cada vez más eficientes y preparen convenientemente a los estudiantes para la vida y el mercado laboral'.

En el seno de la Unión Europea la base de esta internacionalización de la educación se fundamenta en la cooperación entre los estados miembros para la construcción efectiva del Espacio Europeo de Educación Superior, que se ha convertido en un dinamizador de muchas actividades relacionadas con la calidad.

Los ministros de educación europeos, deseosos de afianzar la garantía de la calidad de la enseñanza superior, instaron durante la Conferencia de Bergen ${ }^{2}$ (2005) a la adopción de los Criterios y Directrices para la Garantía de Calidad en el Espacio Europeo de Educación Superior elaborados por la $E N Q A^{3}$. El documento, que se concentra en los tres ciclos de la educación superior descritos en la Declaración de Bolonia y no pretende abarcar ni el ámbito de la investigación ni el de la gestión de la institución, hace una mención explícita a las bibliotecas en el apartado dedicado a recursos de aprendizaje y apoyo para el estudiante y establece que "las instituciones deben controlar, revisar y mejorar, de manera rutinaria, la efectividad de los servicios de apoyo para sus estudiantes".

La adopción de un sistema de garantía de la calidad es una decisión inaplazable. ¿Cómo se plantean las universidades españolas el control, la revisión y la mejora de la efectividad de sus bibliotecas?

\section{Primero evaluar...}

Evaluación y calidad son términos complementarios. ¿Qué sentido 
Bibliotecas que disponen del Certificado de calidad de los servicios de biblioteca de las universidades

\section{3}

- Universidad Autónoma de Barcelona

- Universidad Carlos III de Madrid

- Universidad de Barcelona

- Universidad de La Rioja

- Universidad de Lleida

- Universidad Politécnica de Cataluña

- Universidad Pompeu Fabra

\section{5}

- Universidad Autónoma de Madrid

- Universidad de Burgos

- Universidad de Cádiz

- Universidad de Cantabria

- Universidad de Girona

- Universidad de Granada

- Universidad de las Islas Baleares

- Universidad de Navarra

- Universidad de Salamanca

- Universidad Jaume I

- Universidad Rey Juan Carlos

- Universidad Rovira i Virgili

\section{6}

- Universidad Complutense de Madrid

- Universidad de Alicante

- Universidad de Almería

- Universidad de Málaga

- Universidad de Murcia

- Universidad de Sevilla

\section{Bibliotecas que disponen del} Certificado de calidad ISO 9001:2000

\section{0}

- Universidad Autónoma de Barcelona

\section{4}

- Universidad Alfonso X

- Universidad Jaume I

\section{5}

- Universidad de Deusto

- Universidad de las Islas Baleares

\section{6}

- Universidad de Vigo

- Universidad de Almería ${ }^{2}$

2007

- Universidad de Granada

1. La Universidad Alfonso $X$, dispone de un sistema de calidad certificado que alcanza diferentes actividades y servicios de la institución e incluye la biblioteca.

2. La biblioteca de la Universidad de Almería certificó previamente, en 2004, el proceso de préstamo interbibliotecario.

Una de las bibliotecas de la Universidad de Zaragoza, la Hypatia del Campus Actur, dispone de la certificación de su sistema de calidad desde 2006.

\section{Bibliotecas que disponen del Sello CEG excelencia europea-EFQM}

\section{6}

- Universidad Católica San Antonio de Murcia (Sello 200+)

- Universidad de Cádiz (Sello 400+)

La Universidad Oberta de Catalunya dispone globalmente del Sello CEG de excelencia europea $500+$.

Bibliotecas que disponen de premios autonómicos a la calidad (basados en EFQM)

\section{1}

- Universitat de les Illes Balears (Diploma del Premi balear d'excel-lència en la gestió)

\section{6}

- Universidad de la Rioja (Diploma de Compromiso con la excelencia) 
tiene evaluar si no tenemos el propósito de mejorar los puntos débiles detectados? ¿y cómo sabremos que trabajamos con calidad si no nos sometemos a evaluaciones periódicas que nos lo confirmen y nos ayuden a definir nuevas líneas de mejora?

No fue hasta muy a finales de los años 90 que se realizaron los primeros procesos formales de evaluación de bibliotecas universitarias (autoevaluación, informe externo, informe final), enmarcados dentro del I Plan Nacional de Evaluación de la Calidad de las Universidades (Pnecu) promovido por el Consejo de Universidades. A partir de 2000 , poco a poco, las evaluaciones institucionales se van haciendo más frecuentes. Las agencias de evaluación autonómicas han jugado un papel importante, como también, obviamente, Aneca, que en 2005 inició un programa específico de evaluación del servicio de biblioteca. Sin embargo a finales de 2006 todavía existían bibliotecas universitarias que no habían pasado por este ejercicio de reflexión y transparencia institucional que representa un proceso de evaluación, mientras otras ya han concluido o se hallan en proceso de finalizar su segundo proceso formal de evaluación.

\section{"Un proceso de evaluación formal ha de tener como consecuencia inmediata la elaboración y ejecución de un plan de mejoras"}

\section{...y después certificar}

Un proceso de evaluación formal ha de tener como consecuencia inmediata la elaboración y ejecución de un plan de mejoras. Si el diagnóstico ha sido razonablemente favorable porque la biblioteca está adecuadamente posicionada en la vía de la calidad, ésta puede plan- tearse dar un paso más y buscar el reconocimiento de su buen hacer mediante algún tipo de certificación. Las bibliotecas universitarias españolas han seguido, hasta este momento, cuatro vías para lograr el reconocimiento externo de la calidad de su gestión.

\section{Certificado de calidad de los} servicios de biblioteca de las universidades

En 2003 se creó un programa específico para promover el interés en la mejora de la calidad. La Certificación de la calidad de servicios de biblioteca es un proceso que impulsa la evaluación continua y la mejora de los servicios. Las certificaciones aportan además el reconocimiento público de los esfuerzos realizados en temas de calidad por parte de los centros que las consiguen. Las bibliotecas candidatas son evaluadas por un comité de Aneca y aquéllas que obtienen la certificación de calidad pueden optar a subvenciones que se deben utilizar para mejorar sus servicios en base a la cofinanciación.

La vigencia de las certificaciones se establece en 3 años a partir de la fecha de resolución. Hasta el momento han existido 3 convocatorias con periodicidad anual, obteniendo la certificación un total de 25 bibliotecas universitarias. Se han destinado hasta 2.640.339 $€$ en régimen de cofinanciación a proyectos de mejora de los servicios bibliotecarios que sirvan para afrontar el nuevo modelo de biblioteca como centro de recursos para el apoyo a la docencia, el aprendizaje y la investigación, fomentar la innovación en servicios y reforzar la cooperación bibliotecaria. La existencia de estas ayudas, asociadas a esta certificación, ejerce un poderoso reclamo.

La última de estas convocatorias de la Secretaría de Estado de Universidades e Investigación data de octubre de 2005, de manera que nos encontramos ante la interrupción de este programa o ante una larga demora en vistas, tal vez, a su remodelación. Debido a ello, los certificados de calidad de las 7 primeras bibliotecas en obtenerlos (Resolución de 14 de octubre de 2003) se hallan ya caducados y sin posibilidades de renovación mientras no aparezca una nueva convocatoria. Los centros que no superaron alguno de los requisitos en las anteriores ocasiones, pero que han efectuado las mejoras necesarias para estar en condiciones de optar con éxito a la certificación, se encuentran también a la espera de la aparición de una nueva convocatoria. Y obviamente también se encuentran en esta situación los que no habían podido concurrir hasta el momento por no haber finalizado un proceso de evaluación formal o no disponer de certificación ISO 9001 vigente y que ahora se hallan ya en condiciones de poder optar a la certificación.

\section{Certificado de calidad ISO} 9001:2000

Algunas bibliotecas universitarias han decidido certificar su sistema de gestión de la calidad empleando la norma ISO 9001:20004. En España, a principios de 2007, existen siete sistemas de biblioteca universitaria que disponen de esta certificación. Este número puede ampliarse a ocho si tenemos en cuenta una cuya universidad ha obtenido la certificación global de su sistema de gestión de la calidad.

Organizaciones educativas de diferentes países han depositado en los últimos años la confianza en los beneficios que comporta la implementación y certificación de sistemas de gestión de la calidad basados en la norma ISO 9001:2000. Sin ser numerosas, son cada vez más las instituciones de educación superior europeas que la aplican para asegurar, principalmente, la calidad de los servicios, y no tanto la del proceso de enseñanza-aprendizaje.

Para Karapetrovic ${ }^{5}$ es evidente la relación causa-efecto entre la cer- 
tificación de instituciones de educación superior y la presión gubernamental, relacionando los beneficios que se desprenden de la aplicación de la norma entre los que destacan:

- La documentación mejora el entendimiento entre el personal académico e investigador y el de la administración.

- Los problemas de calidad pueden ser identificados, corregidos y prevenidos, pudiendo realizarse mejoras sistemáticas.

- Las auditorías internas permiten resolver problemas prácticos.

- Las auditorías externas proveen de un punto de vista exterior independiente, a menudo ventajoso para la mejora de la calidad.

En todo caso, es importante tener en cuenta que el uso de la $I S O$ 9001 no se restringe a las instituciones certificadas y los principios de gestión de la calidad establecidos por esta norma pueden ser usados para desarrollar sistemas efectivos de autoevaluación y aseguramiento de la calidad.

\section{Sello CEG de excelencia eu- ropea}

El Club Excelencia en Gestión $(C E G)$ es una asociación sin ánimo de lucro que tiene como objetivo y misión promover la implantación en España de sistemas de gestión de calidad total, basados en el modelo creado por la EFQM. Utiliza herramientas de autoevaluación para diagnóstico de la gestión, que califica en puntos $E F Q M$, lo que permite comparar el nivel de excelencia con otras organizaciones.

Recientemente ha firmado un convenio con Aneca, en base al cual, ésta ha iniciado un programa de evaluación de servicios universitarios y unidades de gestión ${ }^{6}$ basado en este esquema, que parte de la autoevaluación y define los parámetros que hay que tener presentes para evaluar el grado de madurez del sistema de gestión de la organización. En función del resultado de la autoevaluación, el servicio valora la conveniencia de optar o no a los sellos de calidad, con diferentes niveles de excelencia. Una puntuación superior a 200, junto a la elaboración de un plan de mejora e implantación de al menos dos acciones del mismo dan la posibilidad de optar al sello de bronce. Al superar los 400 o 500 puntos y la elaboración de una memoria, se posibilita optar al sello de plata u oro respectivamente.

Los procesos de evaluación de bibliotecas también están siendo llevados a cabo siguiendo cada vez más el modelo EFQM. A la vista del convenio Aneca-CEG, ¿la obtención de sellos de calidad debe interpretarse como el paso lógico a seguir también por las bibliotecas? La demora en la aparición de una nueva convocatoria del Certificado de la calidad de servicios de biblioteca parecería que apunta en esa línea.

Por el momento, al menos una biblioteca universitaria dispone del Diploma CEG Compromiso hacia la excelencia $200+$ y otra ha obtenido el Sello de plata CEG de excelencia europea $400+$.

4. Premios a la excelencia otorgados por las comunidades autónomas

La mayoría de autonomías dispone de algún tipo de premio o reconocimiento de la calidad con la que trabajan sus empresas e instituciones. Las convocatorias suelen mencionar el interés del gobierno autonómico en fomentar la dinámica del modelo EFQM entre el empresariado y la administración de la comunidad como una manera de mejorar la calidad y competitividad de las mismas. Al menos dos bibliotecas universitarias disponen de diplomas de excelencia otorgados por su comunidad.

\section{Sobre todo, consolidar}

La situación actual de la implantación de la calidad en las bibliotecas universitarias nos permite clasificarlas en tres grandes gru$\operatorname{pos}^{7}$ :

- Uno reducido, pero creciente, con sistemas de gestión de la calidad plenamente consolidados que incluye algunos centros que disponen de más de un sello o certificado de calidad y/o que ya han completado su segundo proceso de evaluación.

- Un segundo (más amplio) con sistemas de gestión de la calidad en fase avanzada de consolidación, después de la realización de una primera evaluación institucional y, en muchos casos, disponiendo también de certificación.

- Un tercer grupo (más heterogéneo) que ha realizado recientemente su primer proceso de evaluación, está realizándolo o no lo ha iniciado aún.

Existe un margen de voluntariedad en la participación en este tipo de actuaciones, en el sentido de que las bibliotecas se someten a este control cuando así lo establecen las estrategias y calendarios institucionales.

Un tercio de las bibliotecas universitarias españolas dispone de algún tipo de certificación de la calidad y se constata la aparición de una cierta "multicertificación"

\section{"1/3 de las bibliotecas universitarias españolas dispone de algún tipo de certificación de la calidad y se constata la aparición de una cierta 'multicertificación"”}


en el panorama español, como se dio a principios de los años 90 en el Reino Unido. No podemos descartar que otras certificaciones relacionadas con la calidad y las buenas prácticas atraigan también el interés de las bibliotecas y de sus universidades (por ejemplo la certificación ISO 20000, dedicada a sistemas de gestión de los servicios TIC).

Sin entrar a valorar la conveniencia o necesidad de obtener una o varias certificaciones formales lo importante es que el sistema de gestión de la calidad exista y, ya puestos, mejor si sigue un modelo razonablemente conocido y de eficacia probada que ayude a normalizar los procedimientos de trabajo, mejore la documentación de los procesos, defina claramente las responsabilidades, reduzca la improvisación, facilite la recogida de datos para la gestión, promueva la evaluación continua y la innovación, dote de transparencia a la gestión y, en definitiva, ayude a mejorar la eficacia de los servicios que ofrece la biblioteca.

Más allá de la disponibilidad de sellos, diplomas y certificaciones es crucial construir un sistema donde primen factores encaminados a la mejora global y a la cooperación en el seno de la universidad, en el que la estrategia de calidad de la biblioteca se halle completamente alineada con la estrategia de calidad de la institución. Pero ello no siempre es posible. ¿Qué ocurre si la universi- dad no dispone de una política de calidad clara y bien comunicada ni mucho menos de un sistema general de garantía de la calidad? El entorno institucional puede actuar, aunque sea de manera no premeditada, como freno a la mejora de la calidad del servicio de biblioteca.

\section{"Los sistemas de gestión de la calidad no son una finalidad, sino un medio para el desarrollo de una dinámica de mejora continua dentro de la biblioteca"}

Un sistema de calidad no es un artefacto milagroso, ni los sellos y certificados se mantienen sin esfuerzo y sin constancia. No debemos perder de vista el hecho de que los sistemas de gestión de la calidad no son una finalidad, sino un medio para el desarrollo de una dinámica de mejora continua dentro de la biblioteca. Queda poco tiempo y mucho trabajo por hacer en el camino hacia el Espacio Europeo de Educación Superior y sin la sinergia de la cooperación, también en temas de calidad, el trayecto se presenta difícil.

\section{Notas}

1. Lenn, Marjorie Peace. "International linkages and quality assurance: a shifting paradigm".
En: International conference for quality assurance agencies in higher education. International developments in assuring quality in higher education: selected papers from an international conference. London: Falmer Press, 1994, pp. 127-133.

2. Comunicado de la Conferencia de Ministros Europeos responsables de educación superior. Bergen, 19-20 de Mayo de 2005. El Espacio Europeo de Educación Superior-Alcanzando las metas. Consultado en: 17-05-07.

http://www.crue.org/espaeuro/lastdocs/ComunicadoBergen.pdf

3. Criterios y directrices para la garantía de calidad en el Espacio Europeo de Educación Superior elaborado por la European Association for Quality Assurance in Higher Education (ENQA). Consultado en: 23-06-07.

http://www.aneca.es/active/docs/enqa_criteriosydirectrices_261005.pdf

4. Balagué Mola, Núria. Les normes de qualitat ISO 9000 a les biblioteques d'institucions d'educació superior. Barcelona: Universitat de Barcelona, 2007. Consultado en: 17-05-07.

http://www.tesisenred.net/TDX-0423107123746/index_cs.html

5. Karapetrovic, Stanislav. "ISO 9000 quality system development for engineering schools: why and how should we do it?". En: International conference on engineering education, 2001. Consultado en: 17-05-07.

http://www.ineer.org/Events/ICEE2001/Proceedings/papers/134.pdf

6. Aneca. Evaluación de servicios universitarios $y$ unidades de gestión. Convenio Aneca-CEG. Consulta: 23-06-07.

http://www.aneca.es/active/docs/evalserv_convenioclub.pdf

7. Pinto, María; Balagué, Núria; Anglada, Lluís. "Evaluación y calidad en las bibliotecas universitarias: experiencias españolas entre 1994-2006". En: Revista española de documentación científica, 2007, v. 30, n. 3, pp. 360-379.

Núria Balagué, Servei de Biblioteques, Universitat Autònoma de Barcelona.

nuria.balague@uab.es

http://bd.ub.es/pub/balague/

\section{Versión online de EPI}

El profesional de la información puede consultarse online gratuitamente desde su inicio en 1992 hasta el penúltimo año

(el acceso al año en curso está reservado a los suscriptores).

Más información en:

http://www.elprofesionaldelainformacion.com/contenidos.html 


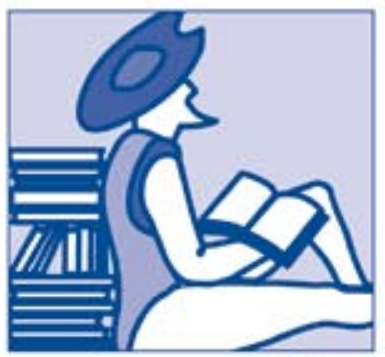

FUNDACIÓN Alonso Quijano

\section{¿qué hacemos?}

Fomento de la lectura con menores hospitalizados.

Actividades de Formación.

Revista Mi Biblioteca.

Cooperación Internacional.

Recursos sobre lectura en la web.

\section{¿quieres colaborar?}

Hazte socio/a y recibirás estos dos libros de regalo*.

\section{Cuota minima: $20 €$ al año.}
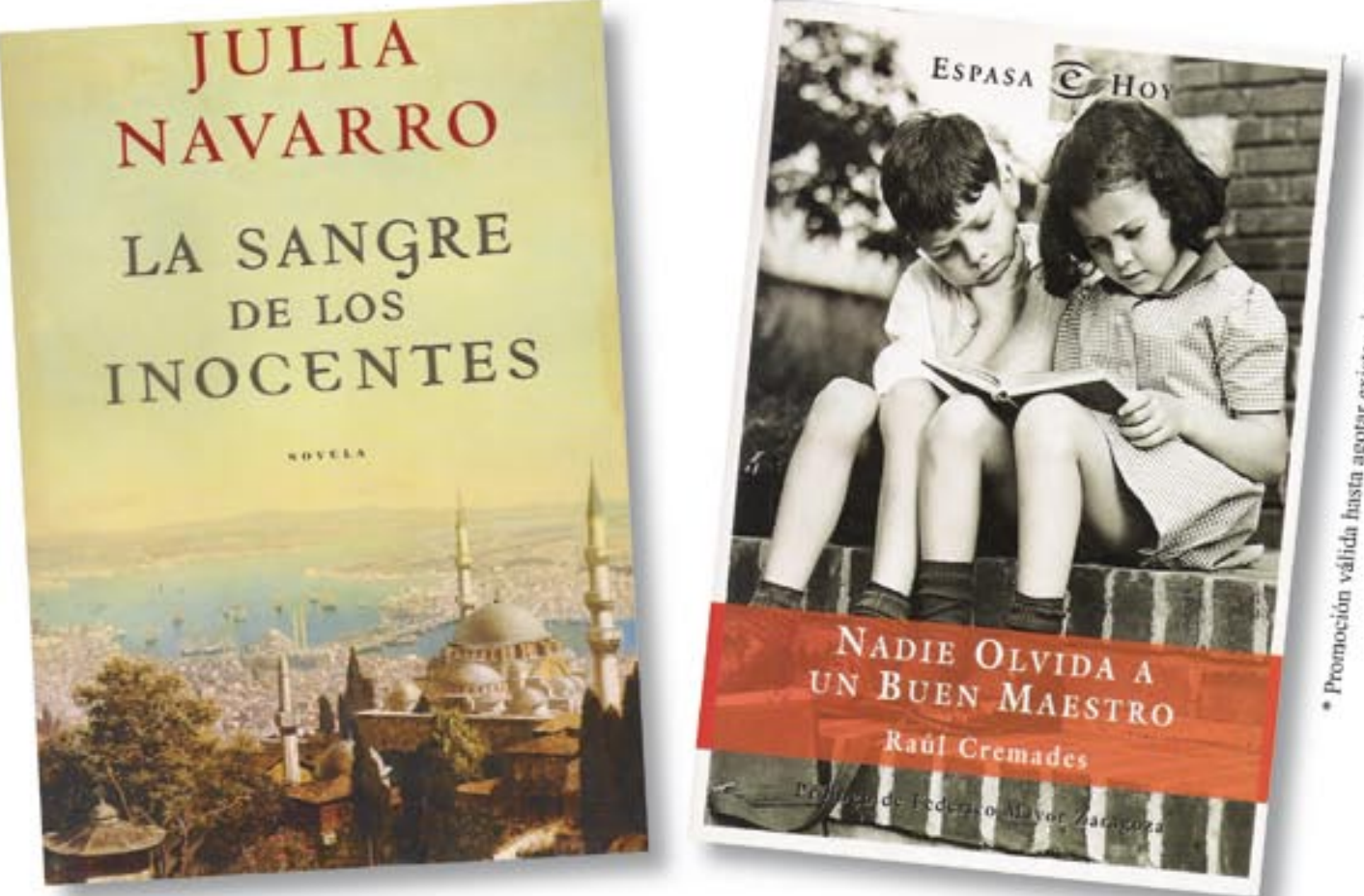

Puedes hacerlo por teléfono: 902362869 - 952235405 o a través de nuestra web: www.alonsoquijano.org

\section{Asóciate y disfruta de estas ventajas:}

- Regalo de un libro cada año como agradecimiento por la colaboración con la Fundación.

- Información sobre las actividades de la Fundación y participación en sorteos y promociones.

- Descuentos en suscripciones a revistas del sector.
- Descuentos en los cursos y otras actividades de formación organizadas por la Fundación.

- Regalo del Calendario de la Lectura que la Fundación publica cada año.

- Ventajas fiscales según la legislación vigente sobre mecenazgo. 